Rev. Int. Contam. Ambie. 34 (4) 737-749, 2018

DOI: $10.20937 /$ RICA.2018.34.04.14

\title{
CADMIO, TALIO Y VANADIO AFECTAN DIFERENCIALMENTE LA GERMINACIÓN Y CRECIMIENTO INICIAL DE TRES VARIEDADES DE CHILE
}

\author{
María de la Luz BUENDÍA-VALVERDE, Libia Iris TREJO-TÉLLEZ*, Tarsicio CORONA-TORRES \\ y Víctor Heber AGUILAR-RINCÓN
}

Colegio de Postgraduados, campus Montecillo, km 36.5 carretera México-Texcoco, 56230 Texcoco, Estado de México, México

*Autora para correspondencia: tlibia@colpos.mx

(Recibido julio 2017; aceptado abril 2018)

Palabras clave: Capsicum annuum, metales pesados, contaminación, fitotoxicidad, bioestimulación

\section{RESUMEN}

Se evaluaron de forma independiente los efectos de los metales cadmio $(\mathrm{Cd})$, talio $(\mathrm{Tl})$ y vanadio (V) a concentraciones de 0,25 y $50 \mu \mathrm{M}$ cada uno, en indicadores de germinación y crecimiento inicial en tres variedades de chile: jalapeño, poblano y serrano. Las semillas fueron tratadas durante 15 días y se cuantificó el número de semillas germinadas en intervalos de $24 \mathrm{~h}$ para la obtención del porcentaje de germinación (PG). Al final de este periodo se determinaron el porcentaje relativo de germinación (PRG), las longitudes de vástagos (LV) y raíces (LR), el crecimiento relativo de radícula (CRR), el índice de germinación (IG) y el coeficiente de velocidad de germinación (CVG). Los tratamientos no afectaron al PG ni al PRG. Los principales efectos de los factores de estudio se observaron en indicadores relacionados con el crecimiento inicial. La tolerancia de las variedades de chile evaluadas a los metales en estudio fue, en orden decreciente, jalapeño $>$ poblano $>$ serrano. El Tl en particular fue altamente fitotóxico en la variedad serrano; por el contrario, el Tl incrementó la LV en las variedades jalapeño y poblano. El Cd estimuló la LV del chile poblano, en tanto que el V estimuló el CVG en el chile jalapeño y la LV del chile poblano. Se concluye que el Cd, Tl y V producen efectos diferenciales entre genotipos, mismos que son dependientes de la variedad de chile probada, la concentración del metal evaluado y la fase de desarrollo (germinación o crecimiento inicial).

Key words: Capsicum annuum, heavy metals, pollution, phytotoxicity, biostimulation

\begin{abstract}
The effects of the metals cadmium $(\mathrm{Cd})$, thallium $(\mathrm{Tl})$ and vanadium $(\mathrm{V})$ in concentrations of 0,25 and $50 \mu \mathrm{M}$ each were evaluated independently in indicators of germination and initial growth in three varieties of chili pepper: jalapeño, poblano, and serrano. The seeds were treated for 15 days, and the number of germinated seeds was quantified in 24-h intervals to obtain the percentage of germination (PG). At the end of this period, the relative percentage of germination (RPG), lengths of shoots (LS) and roots (LR), relative radicle growth (RRG), germination index (GI) and the coefficient of germination speed (CGS) were evaluated. The PG and the RPG were not affected by the treatments.
\end{abstract}


The main effects of the study factors were observed in indicators related to the initial plant growth. The tolerance of the varieties to the metals under study is, in decreasing order, jalapeño $>$ poblano $>$ serrano. $\mathrm{Tl}$ in particular was highly phytotoxic in the serrano variety. Conversely, $\mathrm{Tl}$ increased the LS in the jalapeño and poblano varieties. Cd stimulated the LS of poblano, while V stimulated the CGS in jalapeño, and the LS of the poblano pepper. It is concluded that $\mathrm{Cd}, \mathrm{Tl}$, and $\mathrm{V}$ produce differential effects on chili peppers, which are dependent on the genotype tested, the concentration of the metal evaluated and the developmental phase (germination or initial growth).

\section{INTRODUCCIÓN}

Los metales pesados son ampliamente utilizados tanto en actividades domésticas como agrícolas, médicas, industriales y tecnológicas, lo que ha conducido a una extensa distribución de estos elementos en el ambiente (Tchounwou et al. 2012) y provocado la contaminación de algunos suelos y cuerpos de agua (Tangahu et al. 2011). Si bien algunos de los metales pesados no tienen funciones biológicas conocidas, otros pueden alterar el metabolismo tanto en células animales como en vegetales (Tangahu et al. 2011). Aun a bajas concentraciones, el suministro de metales no esenciales puede modificar los procesos de germinación y crecimiento de plantas superiores (Navarro et al. 2007, Tangahu et al. 2011).

Los metales pesados acumulados en el suelo tienen efectos diferenciales en la producción de cultivos, lo cual depende del genotipo y fase del desarrollo, entre otros factores, siendo de suma importancia la germinación como fase inicial del proceso productivo. La germinación se define como el surgimiento y desarrollo del embrión de la semilla para dar lugar a una nueva plántula. El proceso inicia cuando la semilla que se encuentra en estado quiescente (inactivo) absorbe el agua necesaria para que suceda la rehidratación de proteínas y orgánulos celulares que activarán el metabolismo, en tanto que la síntesis de ácidos nucleicos y proteínas iniciarán la actividad enzimática que finalmente estimulará la emergencia de la radícula (Herrera et al. 2006).

En semillas de Tagetes erecta, Salvia splendens y Abelmoschus manihot la aplicación de $50 \mathrm{mg} \mathrm{Cd} / \mathrm{L}$ no afectó la germinación, aunque inhibió el crecimiento inicial y el peso en fresco de la raíz (Wang y Zhou 2005). De igual forma, disminuyó el crecimiento de plántulas de arroz (Oryza sativa) tratadas con $1 \mu \mathrm{M}$ $\mathrm{Cd}$, aunque a concentraciones menores a $1 \mu \mathrm{M} \mathrm{Cd}$ se observó un efecto estimulante en la fisiología de la planta (Aina et al. 2007).

La alta bioacumulación de talio (Tl) $(221 \mathrm{mg} / \mathrm{kg})$ en plántulas de Lemna minor ocasionada por la exposición a $2 \mu \mathrm{M}$ de acetato de talio inhibió su crecimiento (Babić et al. 2009). En lechuga (Lactuca sativa), la aplicación de $0.5 \mathrm{mg} \mathrm{Tl} / \mathrm{L}$ de solución nutritiva redujo la longitud de la radícula en $67 \%$ en comparación con el testigo. En plantas de nabo (Brassica rapa) tratadas con $2.5 \mathrm{mg} \mathrm{Tl} / \mathrm{L}$, se redujo la longitud de la radícula en 57 \%. En mijo (Panicum miliaceum), la aplicación de $5 \mathrm{mg} \mathrm{Tl} / \mathrm{L}$ redujo la longitud de la radícula en $37 \%$ (Carlson et al. 1991). Por el contrario, semillas de arroz expuestas a dosis de $\mathrm{Tl}$ de 0.1 a $10 \mathrm{mg} / \mathrm{L}$ no mostraron cambios significativos en la germinación, aunque a partir de $5 \mathrm{mg} / \mathrm{L}$ se redujo la longitud de radícula (Yao et al. 2008). En Cannabis sativa cv. Carmagnola y Ferimon, la aplicación de Tl a 15 y $30 \mathrm{mg} / \mathrm{kg}$ de suelo redujo la germinación y ocasionó amarillamiento y necrosis de hojas pequeñas y crecimiento más lento que el testigo (Di Candito et al. 2004).

La aplicación de vanadio (V) en el rango de 50 a $100 \mu \mathrm{g} / \mathrm{mL}$ disminuyó el porcentaje de germinación en soya (Glycine max), trigo (Triticum aestivum), cebada (Hordeum vulgare) y arroz, y redujo significativamente la biomasa total de las plántulas (Tham et al. 2001). Así también, concentraciones de V de $2.5 \mathrm{mg} / \mathrm{L}$ disminuyeron la longitud de radícula en nabo en $49 \%$, en col (Brassica oleracea var. capitata) en $42 \%$ y en lechuga en $27 \%$ (Carlson et al. 1991).

Los bioensayos de germinación de semillas en presencia de metales pesados pueden ser un valioso indicador de la existencia de efectos fitotóxicos (Iannacone y Alvariño 2005). El proceso de germinación es una etapa de gran sensibilidad en que la presencia de cualquier compuesto tóxico puede alterar la supervivencia y el desarrollo normal de la planta. Además, es necesario evaluar el crecimiento y desarrollo de la radícula y del hipocótilo, ya que éstos permiten observar efectos inhibitorios o de retraso que pueden ocurrir sin que haya una alteración durante la germinación y que proporcionan información complementaria durante el crecimiento inicial de la plántula después de la germinación (Sobrero y Ronco 2004). 
Entre los cultivos de mayor importancia en México se encuentra el chile (Capsicum annuum), cuya producción anual en 2015 fue de 2.2 millones de toneladas de fruto fresco. Del total de esta producción, se comercializaron cerca de 900 mil toneladas de chiles frescos, secos y en preparaciones en mercados de Canadá, Japón, Estados Unidos, Alemania y Gran Bretaña. Los tres principales estados productores de este cultivo son Chihuahua, Sinaloa y Zacatecas, con una producción de 562, 556 y 348 mil toneladas por año, respectivamente (SAGARPA 2015). En estos estados no existe un control de contaminantes en los campos agrícolas, aun cuando prevalecen reportes de metales pesados en diversas zonas, provenientes de desechos de la industria minera y de aguas residuales empleadas para el riego (Maldonado et al. 2008, Frías-Espericueta et al. 2009, González-Dávila et al. 2012).

Entre los metales reportados tanto en suelo como en agua, se encuentran cadmio (Cd), Tl y V. Por ejemplo, en suelos mineros de Zacatecas el Cd presenta una concentración media de $6.9 \mathrm{mg} / \mathrm{kg}$, siendo chile $\mathrm{y}$ avena los cultivos con mayor acumulación de este elemento, con valores de 6.1 y $5.6 \mathrm{mg} / \mathrm{kg}$, respectivamente (Garay-Hernández et al. 2012). Respecto al Tl, su estudio no ha recibido suficiente atención debido, en parte, a la dificultad de medición dadas sus concentraciones relativamente bajas y a la falta de materiales de referencia ambiental que tengan valores certificados (Queirolo et al. 2009). Para México, el límite de concentración de $\mathrm{Tl}$ en suelos agrícolas es de $5.2 \mathrm{mg} / \mathrm{kg}$ (SEMARNAT 2007). En suelos urbanos de la ciudad de Chihuahua, el V se encuentra en concentraciones que varían de 3 a $230 \mathrm{mg} / \mathrm{kg}$, y el límite permitido en suelos agrícolas es de $78 \mathrm{mg} / \mathrm{kg}$ (Alcalá et al. 2009). En Sinaloa se analizaron concentraciones de metales pesados en lagunas costeras, y se encontraron niveles de Cd de 3.64 a $6.91 \mathrm{mg} / \mathrm{g}$, lo cual representa un posible riesgo para la salud humana y el ambiente (Frías-Espericueta et al. 2009).

En la producción de plántulas de cultivos hortícolas como el chile, sobre todo en sistemas de agricultura protegida, además del agua de riego y el suelo es común el uso de otros sustratos como la turba de musgo (Sphagnum capillifolium). La turba es un material de bajo costo con alta capacidad de adsorción de metales pesados. Dada su acidez natural ( $\mathrm{pH}$ entre 4.5 y 6.0 ), la turba puede disminuir el $\mathrm{pH}$ del suelo cuando se mezcla con éste, lo que ocasiona mayor disponibilidad de metales pesados para las raíces de las plantas (Lee et al. 2015, Kumar 2017). De hecho, en el musgo $S$. capillifolium se ha reportado la presencia de $\mathrm{V}$ y $\mathrm{Cd}$ en concentraciones promedio de 1.04 y $3.48 \mathrm{mg} / \mathrm{kg}$ de peso seco, respectivamente (Meyer et al. 2015).

El objetivo de este estudio fue evaluar los efectos de los metales pesados no esenciales $\mathrm{Cd}, \mathrm{Tl}$ y $\mathrm{V}$, en parámetros de germinación y crecimiento inicial de plántulas de tres variedades de chile (Capsicum annuum).

\section{MATERIALES Y MÉTODOS}

\section{Material vegetal}

Se utilizaron semillas híbridas de chile (Capsicum annuum) de las siguientes variedades: jalapeño emperador NUN 70030, poblano capulín y serrano coloso.

\section{Desinfestación de semillas}

Para eliminar la posible presencia de patógenos, las semillas se incubaron en una solución de hipoclorito de sodio al $2 \%(0.33 \mathrm{~mL} / \mathrm{L})$ durante $15 \mathrm{~min}$, y posteriormente se hicieron seis enjuagues con agua destilada estéril.

\section{Tratamientos y diseño experimental}

Se realizaron nueve ensayos independientes en los que se empleó un diseño experimental completamente al azar. Cada ensayo corresponde a uno de los metales evaluados a tres concentraciones por variedad (Cuadro I). Se utilizaron cloruro de cadmio $\left(\mathrm{CdCl}_{2}\right.$, CAS 10108-64, Sigma Aldrich), acetato de talio $\left(\mathrm{CH}_{3} \mathrm{COOT1}\right.$, CAS 563-68-5, Sigma Aldrich), y metavanadato de amonio $\left(\mathrm{NH}_{4} \mathrm{VO}_{3}, \mathrm{CAS}\right.$ 7803-55-6, Alfa Aesar) como fuentes de Cd, Tl y V, respectivamente.

Cada tratamiento tuvo cuatro repeticiones y la unidad experimental consistió en una caja de Petri con 10 semillas. Las cajas de Petri se distribuyeron de manera aleatoria dentro de una cámara de germinación (Thermo Scientific, modelo $310 \mathrm{M}$, EUA) a $25^{\circ} \mathrm{C}$ en oscuridad (ISTA 2009), donde se mantuvieron debidamente hidratadas con la solución correspondiente al tratamiento asignado (metal y concentración) durante 15 días. Las cajas de Petri de los tratamientos testigo fueron hidratadas con agua destilada.

\section{Variables evaluadas}

El estudio se condujo durante 15 días dado que el rango de germinación reportado para diversas variedades de chile varía de 3 a 12 días, con niveles de germinación máxima de 5 a 7 días (Demir et al. 2008, Aloui et al. 2014, Ayala-Villegas et al. 2014, García-Gaytán et al. 2017). Asimismo, el periodo 
CUADRO I. ENSAYOS CONDUCIDOS CON Cd, Tl y V EN SEMILLAS DE CHILE DE LAS VARIEDADES JALAPEÑO, POBLANO Y SERRANO

\begin{tabular}{|c|c|c|c|c|c|c|c|c|c|}
\hline \multirow[b]{3}{*}{ Jalapeño } & \multicolumn{3}{|c|}{ Experimento 1} & \multicolumn{3}{|c|}{ Experimento 4} & \multicolumn{3}{|c|}{ Experimento 7} \\
\hline & \multicolumn{3}{|c|}{ Cadmio $(\mu \mathrm{M})$} & \multicolumn{3}{|c|}{ Talio $(\mu \mathrm{M})$} & \multicolumn{3}{|c|}{ Vanadio $(\mu \mathrm{M})$} \\
\hline & 0 & 25 & 50 & 0 & 25 & 50 & 0 & 25 & 50 \\
\hline Variedad & \multicolumn{3}{|c|}{ Experimento 2} & \multicolumn{3}{|c|}{ Experimento 5} & \multicolumn{3}{|c|}{ Experimento 8} \\
\hline \multirow{2}{*}{ Poblano } & \multicolumn{3}{|c|}{ Cadmio $(\mu \mathrm{M})$} & \multicolumn{3}{|c|}{ Talio $(\mu \mathrm{M})$} & \multicolumn{3}{|c|}{ Vanadio $(\mu \mathrm{M})$} \\
\hline & 0 & 25 & 50 & 0 & 25 & 50 & 0 & 25 & 50 \\
\hline Variedad & \multicolumn{3}{|c|}{ Experimento 3} & \multicolumn{3}{|c|}{ Experimento 6} & \multicolumn{3}{|c|}{ Experimento 9} \\
\hline \multirow[t]{2}{*}{ Serrano } & \multicolumn{3}{|c|}{ Cadmio $(\mu \mathrm{M})$} & \multicolumn{3}{|c|}{ Talio $(\mu \mathrm{M})$} & \multicolumn{3}{|c|}{ Vanadio $(\mu \mathrm{M})$} \\
\hline & 0 & 25 & 50 & 0 & 25 & 50 & 0 & 25 & 50 \\
\hline
\end{tabular}

de ensayo de 15 días permitió la medición del crecimiento inicial de la plántula.

\section{Porcentaje de germinación (PG)}

Esta variable se midió 15 días después del establecimiento de las semillas y correspondió al valor porcentual real de todas las semillas germinadas durante el ensayo. Se consideró la semilla germinada cuando mostró una radícula de $2 \mathrm{~mm}$ de longitud.

\section{Porcentaje relativo de germinación (PRG)}

Se estimó de acuerdo a la fórmula siguiente (Tiquia 2000):

$P R G=\frac{\begin{array}{c}\text { Semillas germinadas en cada } \\ \text { tratamiento por variedad }\end{array}}{\begin{array}{c}\text { Semillas germinadas en el tratamiento } \\ \text { testigo por variedad }\end{array}} \times 100$

\section{Índice de germinación (IG)}

Se calculó de acuerdo con lo descrito por Tiquia (2000) empleando la fórmula que relaciona el porcentaje relativo de germinación y el crecimiento relativo de la radícula, la cual ha sido empleada en estudios de fitotoxicidad por metales pesados (Walter et al. 2006):

$I G=\frac{P R G \times C R R}{(100)}$

donde $P R G$ es el porcentaje relativo de germinación, variable que fue descrita previamente y $C R R$ el crecimiento relativo de radícula, cuya fórmula de cálculo se describe posteriormente.

\section{Coeficiente de velocidad de germinación (CVG)}

Se calculó con la fórmula descrita por Kader (2005) como sigue:

$$
C V G=\frac{\begin{array}{c}
\text { Número total de semillas } \\
\text { germinadas por caja de Petri }
\end{array}}{A 1 T 1+A 2 T 2+A x T x}
$$

donde $A$ es el número de semillas germinadas, $T$ el tiempo en días correspondiente a $A$ y los números 1 , $2, . . x$ corresponden al número de días desde el inicio (1) hasta la evaluación final.

\section{Longitud de radícula (LR) y longitud de vástago (LV)}

Las longitudes de la radícula y el vástago se midieron con una regla graduada de $20 \mathrm{~cm}$.

\section{Crecimiento relativo de radícula (CRR)}

Se estimó empleando la siguiente fórmula (Tiquia 2000):

$$
C R R=\frac{\begin{array}{c}
\text { Longitud de radiculas } \\
\text { en semillas tratadas }
\end{array}}{\begin{array}{l}
\text { Longitud de radiculas } \\
\text { en semillas del testigo }
\end{array}} \times 100
$$

\section{Análisis estadístico}

Con los resultados obtenidos se realizaron análisis de varianza y pruebas de comparación de medias (diferencia mínima significativa, $\mathrm{p} \leq 0.05$ ), independientes para cada metal y variedad, usando el software SAS (SAS 2011).

\section{RESULTADOS Y DISCUSIÓN}

Los resultados de los tres ensayos se presentan de manera conjunta por variable. De esta manera se pueden evidenciar los efectos de cada uno de los metales en los parámetros evaluados en las tres variedades de chile, de manera independiente.

\section{Porcentaje de germinación (PG)}

En plantas superiores, el $\mathrm{Cd}$ puede inhibir la germinación de semillas y el crecimiento de raíces, e inducir aberraciones cromosómicas y formación 
de micronúcleos (Aslam et al. 2014). Las semillas de chile jalapeño tratadas con $\mathrm{Cd}(25$ y $50 \mu \mathrm{M})$ presentaron una media de porcentaje de germinación de $96.3 \%$, mientras que la del testigo fue de $97.5 \%$ (Cuadro II).

CUADRO II. PORCENTAJES DE GERMINACIÓN DE SEMILLAS DE TRES VARIEDADES DE CHILE TRATADAS CON Cd, Tl Y V

\begin{tabular}{|c|c|c|c|}
\hline \multirow{2}{*}{ Variedad } & \multicolumn{3}{|c|}{$\mathrm{Cd}(\mu \mathrm{M})$} \\
\hline & 0 & 25 & 50 \\
\hline Jalapeño & $97.5 \pm 2.5 \mathrm{a}$ & $97.5 \pm 2.5 \mathrm{a}$ & $95.0 \pm 2.9 \mathrm{a}$ \\
\hline Poblano & $95.0 \pm 2.9 \mathrm{a}$ & $97.5 \pm 2.5 \mathrm{a}$ & $95.0 \pm 2.9 \mathrm{a}$ \\
\hline Serrano & $100.0 \pm 0.0 \mathrm{a}$ & $100.0 \pm 0.0 \mathrm{a}$ & $100.0 \pm 0.0 \mathrm{a}$ \\
\hline \multirow{2}{*}{ Variedad } & \multicolumn{3}{|c|}{$\mathrm{Tl}(\mu \mathrm{M})$} \\
\hline & 0 & 25 & 50 \\
\hline Jalapeño & $97.5 \pm 2.5 \mathrm{a}$ & $90.0 \pm 4.1 \mathrm{a}$ & $95.0 \pm 2.9 \mathrm{a}$ \\
\hline Poblano & $95.0 \pm 2.9 \mathrm{a}$ & $90.0 \pm 3.9 \mathrm{a}$ & $95.0 \pm 2.9 \mathrm{a}$ \\
\hline Serrano & $100.0 \pm 0.0 \mathrm{a}$ & $92.5 \pm 4.8 \mathrm{a}$ & $100.0 \pm 0.0 \mathrm{a}$ \\
\hline \multirow{2}{*}{ Variedad } & \multicolumn{3}{|c|}{$\mathrm{V}(\mu \mathrm{M})$} \\
\hline & 0 & 25 & 50 \\
\hline Jalapeño & $97.5 \pm 2.5 \mathrm{a}$ & $90.0 \pm 4.0 \mathrm{a}$ & $95.0 \pm 2.8 \mathrm{a}$ \\
\hline Poblano & $95.0 \pm 2.9 \mathrm{a}$ & $92.5 \pm 2.5 \mathrm{a}$ & $90.0 \pm 0.0 \mathrm{a}$ \\
\hline Serrano & $100.0 \pm 0.0 \mathrm{a}$ & $95.0 \pm 2.9 \mathrm{a}$ & $100.0 \pm 0.0 \mathrm{a}$ \\
\hline
\end{tabular}

Medias \pm desviación estándar con letras iguales en cada hilera, indican que no existen diferencias estadísticas significativas (diferencia mínima significativa, $\mathrm{p}>0.05$ )

En Capsicum frutescens, la aplicación de Cd a concentraciones de 75 a $600 \mathrm{mg} / \mathrm{kg}$ de suelo ocasionó efectos fitotóxicos (Khan y Moheman 2006). Si bien estas concentraciones son superiores a las aquí evaluadas, debe considerarse que el suelo tiene una capacidad amortiguadora que reduce en gran medida la disponibilidad de este elemento. Asimismo, los efectos negativos del $\mathrm{Cd}$ se han reportado sólo cuando éste se abastece a altas concentraciones, lo que ocasiona estrés oxidativo (Kranner y Colville 2011).

Las semillas de chile poblano tratadas con $\mathrm{Cd}$ tuvieron un promedio de germinación de $96.3 \%$, mientras que en el testigo éste fue de $95 \%$. En el caso del chile serrano, la germinación fue del $100 \%$, tanto en el testigo como con las dos concentraciones de Cd evaluadas (Cuadro II). Entre especies, el efecto del $\mathrm{Cd}$ en la germinación es diferencial. Por ejemplo, al tratar semillas de acelga (Beta vulgaris var. cicla), lechuga y espinaca con $25 \mu \mathrm{M}$ de $\mathrm{Cd}$, se redujo el porcentaje de germinación en 46, 97 y $8 \%$, respectivamente (Bautista et al. 2013). Asimismo, los tratamientos de $10,30,50,70$ y $90 \mu \mathrm{mol} \mathrm{Cd} / \mathrm{L}$ disminuyeron la germinación de semillas de Albizia lebbeck, en comparación con el testigo, lo cual puede atribuirse a una posible degradación de sustancias nutritivas almacenadas en las semillas (Farooqi et al. 2009).

A pesar de no existir diferencias estadísticas significativas, en las tres variedades tratadas con Tl se observaron los menores porcentajes de germinación a la concentración de $25 \mu \mathrm{M}$, con decrementos del orden de 7.7, 5.3 y $7.5 \%$ en las variedades jalapeño, poblano y serrano, respectivamente, en comparación con sus respectivos testigos. Con la dosis alta de $\mathrm{Tl}$ $(50 \mu \mathrm{M})$, el porcentaje de germinación registrado en las variedades poblano y serrano fue el mismo que en sus testigos (Cuadro II). En semillas de arroz, el tratamiento con $\mathrm{Tl}$ en el intervalo $0.1 \mathrm{a} 10 \mathrm{mg} / \mathrm{L}$ no tuvo influencia en la germinación de las semillas (Yao et al. 2008). Infortunadamente, los reportes acerca del efecto del $\mathrm{Tl}$ en la germinación de semillas son escasos en la literatura científica especializada.

En semillas de las tres variedades de chile tratadas con V no se observaron diferencias estadísticas significativas en el porcentaje de germinación (Cuadro II). No obstante, se aprecia la misma tendencia registrada en el tratamiento con $\mathrm{Tl}$ en dos variedades (jalapeño y serrano), dado que el porcentaje de germinación se redujo en mayor proporción con la dosis baja de V. Con $25 \mu \mathrm{M} \mathrm{V}$, la reducción en la germinación fue de $7.5,2.5$ y $5 \%$ en las variedades jalapeño, poblano y serrano, respectivamente, en comparación con los testigos (Cuadro II). Los estudios del efecto del $\mathrm{V}$ en la germinación son escasos y los existentes se han realizado en gran medida en cereales. En centeno (Secale cereale) y trigo se demostró que el tratamiento con $0.1 \mu \mathrm{M}$ de $\mathrm{V}$ afectó ligeramente la germinación de semillas (Kasai et al. 1999). Contrario a lo aquí observado, en arroz, bajos niveles de $\mathrm{V}$ aceleraron la germinación, en tanto que altas dosis la inhibieron (Zhao et al. 2007). Semillas de garbanzo (Cicer arietinum) incubadas durante seis días con soluciones de $\mathrm{NaVO}_{3}$ en concentraciones de 410 a $1640 \mu \mathrm{M}$, mostraron un porcentaje de germinación superior al $99 \%$ (Zhang et al. 2012).

\section{Porcentaje relativo de germinación (PRG)}

Las semillas de chile jalapeño tratadas con $\mathrm{Cd}$ presentaron una media de porcentaje relativo de germinación de $98.7 \%$, mientras que las de poblano y serrano tratadas con este mismo metal presentaron $100 \%$ de germinación relativa. Destaca el hecho 
de que en la variedad poblano, el suministro de 25 $\mu \mathrm{M}$ Cd elevó a $102.6 \%$ el PRG, como se observa en el cuadro III. En trigo, la aplicación de $250 \mu \mathrm{M}$ de Cd disminuyó la germinación en $30 \%$ y en $25 \%$ en las variedades Galaxy y NARC-11, respectivamente (Idrees et al. 2015). También se ha reportado que la aplicación de 1 y $6 \mathrm{mg}$ de $\mathrm{Cd} / \mathrm{L}$ (i.e., 8.9 a $53.4 \mu \mathrm{M}$ Cd) inhiben de 18 a $88 \%$ el porcentaje de germinación en Suaeda salsa, respectivamente (Liu et al. 2012).

CUADRO III. PORCENTAJES RELATIVOS DE GERMINACIÓN DE SEMILLAS DE TRES VARIEDADES DE CHILE TRATADAS CON Cd, Tl Y V

\begin{tabular}{|c|c|c|c|}
\hline \multirow{2}{*}{ Variedad } & \multicolumn{3}{|c|}{$\mathrm{Cd}(\mu \mathrm{M})$} \\
\hline & 0 & 25 & 50 \\
\hline Jalapeño & $100.0 \pm 2.6 \mathrm{a}$ & $100.0 \pm 2.6 \mathrm{a}$ & $97.4 \pm 3.0 \mathrm{a}$ \\
\hline Poblano & $100.0 \pm 3.0 \mathrm{a}$ & $102.6 \pm 2.6 \mathrm{a}$ & $100.0 \pm 3.0 \mathrm{a}$ \\
\hline Serrano & $100.0 \pm 0.0 \mathrm{a}$ & $100.0 \pm 0.0 \mathrm{a}$ & $100.0 \pm 0.0 \mathrm{a}$ \\
\hline \multirow{2}{*}{ Variedad } & \multicolumn{3}{|c|}{$\mathrm{Tl}(\mu \mathrm{M})$} \\
\hline & 0 & 25 & 50 \\
\hline Jalapeño & $100.0 \pm 2.6 \mathrm{a}$ & $92.3 \pm 4.2 \mathrm{a}$ & $94.7 \pm 3.0 \mathrm{a}$ \\
\hline Poblano & $100.0 \pm 3.0 \mathrm{a}$ & $94.7 \pm 4.3 \mathrm{a}$ & $100.0 \pm 3.0 \mathrm{a}$ \\
\hline Serrano & $100.0 \pm 0.0 \mathrm{a}$ & $92.5 \pm 4.8 \mathrm{a}$ & $100.0 \pm 0.0 \mathrm{a}$ \\
\hline \multirow{2}{*}{ Variedad } & \multicolumn{3}{|c|}{$\mathrm{V}(\mu \mathrm{M})$} \\
\hline & 0 & 25 & 50 \\
\hline Jalapeño & $100.0 \pm 2.6 \mathrm{a}$ & $92.3 \pm 4.2 \mathrm{a}$ & $97.4 \pm 3.0 \mathrm{a}$ \\
\hline Poblano & $100.0 \pm 3.0 \mathrm{a}$ & $97.4 \pm 2.6 \mathrm{a}$ & $94.7 \pm 0.0 \mathrm{a}$ \\
\hline Serrano & $100.0 \pm 0.0 \mathrm{a}$ & $95.0 \pm 2.9 \mathrm{a}$ & $100.0 \pm 0.0 \mathrm{a}$ \\
\hline
\end{tabular}

Medias \pm desviación estándar con letras iguales en cada hilera, indican que no existen diferencias estadísticas significativas (diferencia mínima significativa, $\mathrm{p}>0.05$ )

En las tres variedades de chile tratadas con $\mathrm{Tl}$ no se observaron diferencias significativas respecto a sus testigos, aunque se observó un menor porcentaje relativo de germinación en la concentración de $25 \mu \mathrm{M}$, con decrementos de 7.6, 5.2 y 7.5\% en las variedades jalapeño, poblano y serrano. Con la dosis alta de $\mathrm{Tl}$ $(50 \mu \mathrm{M})$, el PRG registrado en las variedades poblano y serrano fue el mismo que en los testigos, aunque para la variedad jalapeño se observó una disminución del $2.5 \%$ respecto al testigo (Cuadro III).

En el caso de las variedades de semillas tratadas con $\mathrm{V}$ no se observaron diferencias estadísticas significativas en el porcentaje relativo de germinación; sin embargo, existió un decremento en esta variable de $7.6,2.6$ y $5.0 \%$ en jalapeño, poblano y serrano, respectivamente, al tratarse con $25 \mu \mathrm{M} \mathrm{V}$. De la misma manera, con la dosis alta de $\mathrm{V}(50 \mu \mathrm{M})$ se redujo el porcentaje relativo de germinación a 2.5 y $5.2 \%$ en las variedades de jalapeño y poblano. Estos resultados evidencian que, de manera general, aun suministrado a bajas concentraciones el $\mathrm{V}$ reduce en mayor proporción el porcentaje relativo de germinación (Cuadro III).

Los estudios del efecto del V en la germinación son escasos, si bien se ha reportado que el tratamiento con $50 \mathrm{mg} \mathrm{V} / \mathrm{mL}$ reduce el porcentaje de germinación de semillas de trigo, cebada, arroz y soya en 52, 65, 4 y $6 \%$, respectivamente. Además, el tratamiento con $100 \mathrm{mg} \mathrm{V} / \mathrm{mL}$ redujo el porcentaje de germinación relativa en $90,85,5$, y $6 \%$, respectivamente (Tham et al. 2001).

\section{Índice de germinación (IG)}

El tratamiento con Cd no afectó de manera significativa el IG de semillas de las variedades jalapeño y serrano; por el contrario, en la variedad poblano este indicador disminuyó de manera significativa cuando las semillas se trataron con este elemento en todas las concentraciones evaluadas (Fig. 1a). El efecto del Cd observado en la variedad poblano es similar al reportado en Suaeda salsa, donde el IG disminuyó significativamente con la dosis de $0.1 \mathrm{mg} \mathrm{Cd} / \mathrm{L}$ (Liu et al. 2012). Asimismo, en semillas de Albizia lebbeck, el índice de vigor mostró una disminución gradual con el aumento de la concentración de $\mathrm{Cd}$ (de 10 a $90 \mu \mathrm{mol} / \mathrm{L}$ ), en comparación con el testigo (Farooqi et al. 2009).

Las semillas de las variedades jalapeño y serrano mostraron susceptibilidad diferencial al Tl. En jalapeño, el tratamiento con $50 \mu \mathrm{M}$ Tl redujo el índice de germinación a $32.7 \%$. En serrano, los tratamientos con 25 y $50 \mu \mathrm{M} \mathrm{Tl}$ ocasionaron índices de germinación de solo 40.7 y $32.3 \%$, respectivamente. Por el contrario, el Tl no afectó el índice de germinación de las semillas de chile poblano (Fig. 1b).

El tratamiento con $\mathrm{V}$ no afectó de manera significativa el IG de las variedades jalapeño y serrano; empero, en ambas variedades se observan tendencias iguales. Por un lado, la concentración alta de V $(50 \mu \mathrm{M})$ ocasionó índices de germinación superiores al $100 \%$ (121 y $116.2 \%$ en jalapeño y serrano, respectivamente). Por el contrario, la concentración baja de $\mathrm{V}$ $(25 \mu \mathrm{M})$ redujo ligeramente los índices de germinación (92 y $96 \%$ en jalapeño y serrano, respectivamente). En la variedad poblano, los índices de germinación fueron reducidos de manera significativa al aplicar 50 y $100 \mu \mathrm{M}$ de $\mathrm{V}$, con valores de 64.8 y $45.1 \%$, respectivamente (Fig. 1c). 
a)

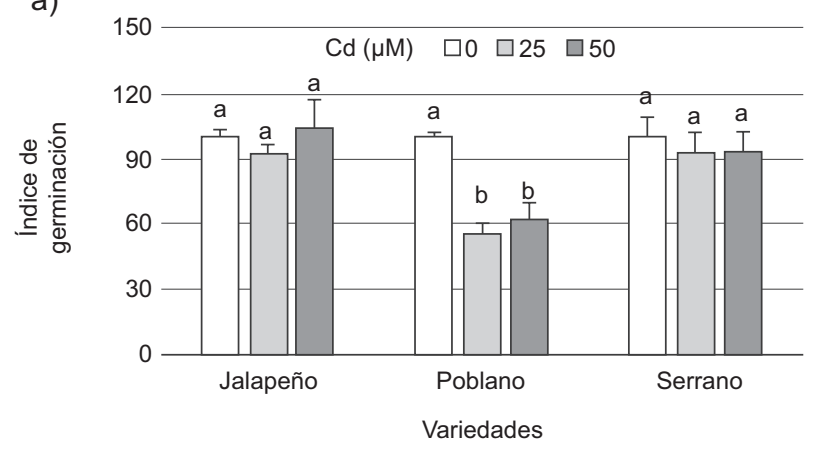

b)

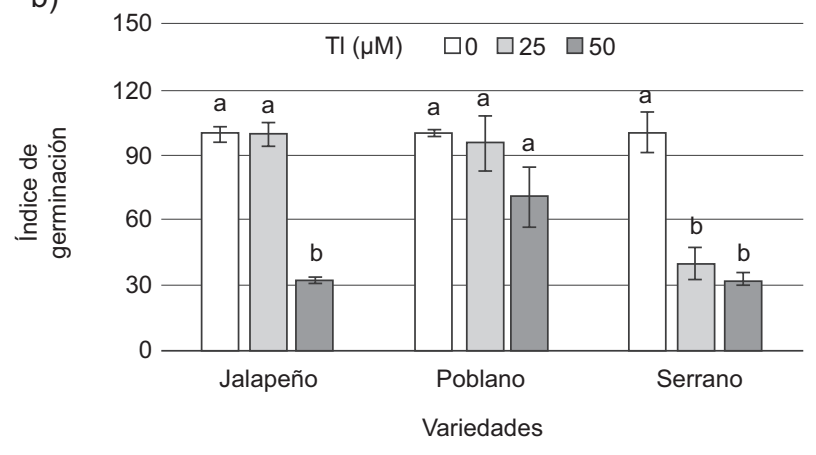

c)

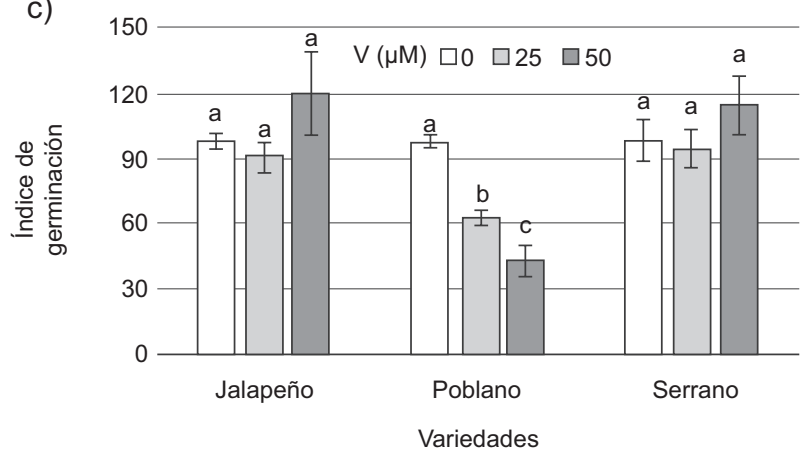

Fig. 1. Índice de germinación (IG) de semillas de tres variedades de chile tratadas con (a) Cd, (b) $\mathrm{Tl}$ y (c) V. Medias \pm desviación estándar con letras distintas en cada variedad por subfigura indican que existen diferencias estadísticas significativas (diferencia mínima significativa, $\mathrm{p} \leq 0.05$ )

El índice de germinación (IG) se utiliza en diversos estudios como indicador de fitotoxicidad. Los valores del IG menores al $50 \%$ indican alta fitotoxicidad, valores de 50 a $80 \%$ sugieren fitotoxicidad moderada, y valores superiores a 80 y menores a $100 \%$ indican que no existe fitotoxicidad. Si el valor del IG excede el $100 \%$, se considera que el material o tratamiento empleado es un fitonutrimento o bien un fitoestimulante (Emino y Warman 2004). Si bien el empleo del índice de germinación fue inicialmente reportado como parámetro útil para determinar la fitotoxicidad de las compostas, este parámetro se ha usado en estudios con otros objetivos, entre ellos la evaluación de la fitotoxicidad por metales. En arroz, la aplicación de $100 \mu \mathrm{M} \mathrm{Cd}$ redujo de manera significativa el índice de germinación, así como el índice de vigor y la longitud de raíces y vástago, respecto al testigo (He et al. 2014). En trigo, la presencia de $\mathrm{Cd}$ afectó el IG, de manera que el metal resultó fitotóxico para esta especie (Ahmad et al. 2012).

En nuestro estudio, el Cd resultó moderadamente fitotóxico en las concentraciones ensayadas en la variedad poblano. El Tl fue altamente fitotóxico en la variedad serrano. El V tuvo efectos diferenciales entre variedades: en jalapeño y serrano actuó como fitoestimulante; por el contrario, en la variedad poblano tuvo efectos moderadamente tóxicos cuando se suministró a $25 \mu \mathrm{M}$, y altamente tóxicos a $50 \mu \mathrm{M}$.

\section{Coeficiente de velocidad de germinación (CVG)}

El Cd afectó de manera significativa al CVG sólo en las semillas de chile serrano. En esta variedad, las concentraciones de 25 y $50 \mu \mathrm{M}$ Cd redujeron el CVG en 19.2 y $18.4 \%$, respectivamente, en comparación con el testigo (Fig. 2a).

Al aplicar dosis bajas de Tl $(25 \mu \mathrm{M})$, el CVG disminuyó en las variedades de jalapeño y serrano en 7.1 y $15.5 \%$ respectivamente, comparado con los testigos de cada variedad. En el caso del suministro de la dosis alta de $\mathrm{Tl}(50 \mu \mathrm{M})$, el CVG disminuyó en 14.2 y $12.1 \%$ en las variedades jalapeño y serrano, respectivamente, en comparación con el testigo y se observó que estas reducciones fueron significativas. En la variedad poblano, el tratamiento con $\mathrm{Tl}$ no afectó el CVG (Fig. 2b).

La aplicación de $\mathrm{V}$ en las variedades de chile evaluadas afectó de manera diferencial al CVG. En la variedad poblano, el V no afectó los CVG de las semillas. En las semillas tratadas con V se observó que a dosis bajas $(25 \mu \mathrm{M})$ el CVG disminuyó en 7.4, 2.4 y $8.5 \%$ en las variedades de jalapeño, poblano y serrano, respectivamente, comparado con sus testigos, aunque estas reducciones no fueron significativas. Por otra parte, con la dosis alta de V $(50 \mu \mathrm{M})$, aumentó aproximadamente en $22 \%$ el CVG de semillas de la variedad jalapeño en comparación con el testigo, y tal aumento fue significativo (Fig. 2c).

La velocidad de emergencia es de suma importancia, dado que define el tiempo de establecimiento en campo de las plántulas provenientes de semilleros o viveros (Sobrevilla-Solis et al. 2013). El CVG es un indicador que permite definir si la germinación de una semilla es lenta o rápida. El CVG varía de 0 a 1 , valores que corresponden a la no germinación y 
a)

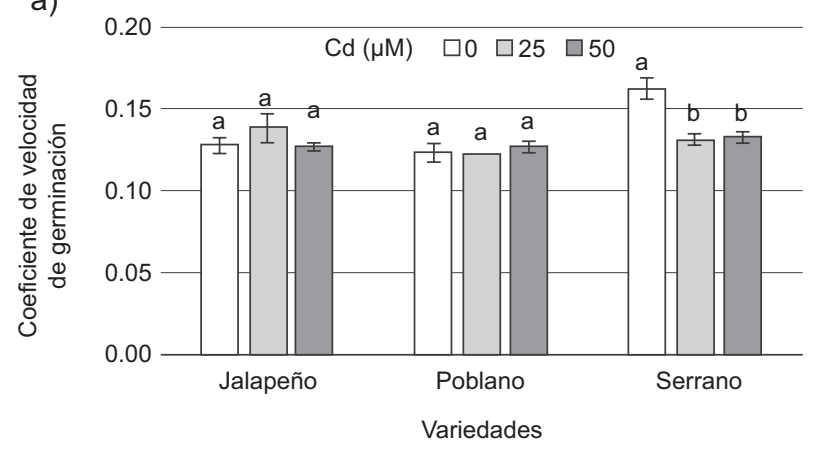

b)

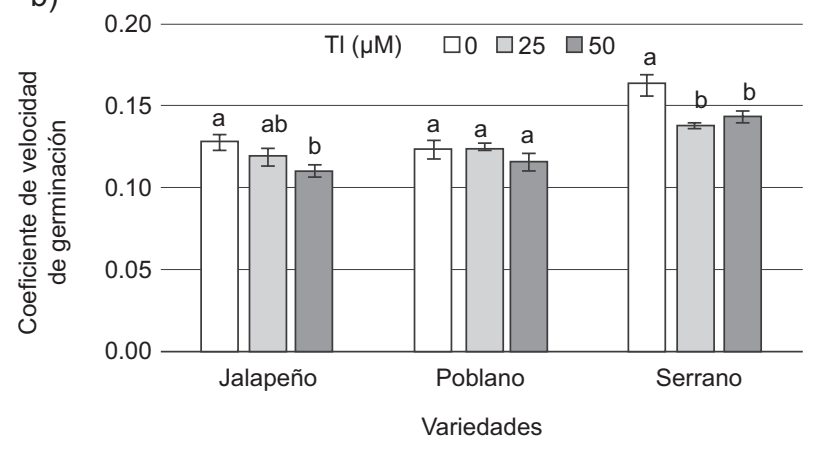

c)

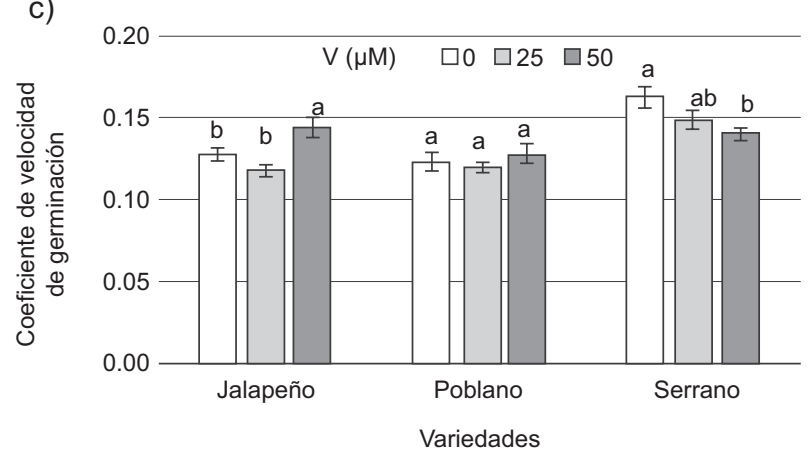

Fig. 2. Coeficiente de velocidad de germinación (CVG) de semillas de tres variedades de chile tratadas con (a) Cd, (b) Tl y (c) V. Medias \pm desviación estándar con letras distintas en cada variedad por subfigura indican que existen diferencias estadísticas significativas (diferencia mínima significativa, $\mathrm{p} \leq 0.05$ )

a la rápida germinación, respectivamente (Sánchez et al. 2015). Asimismo, es importante considerar que el CVG es el recíproco del tiempo promedio de germinación (Ranal y García 2006), por tanto es independiente del porcentaje de germinación final (Kader 2005). En este sentido se concluye que en las semillas testigo (no expuestas a $\mathrm{Cd}, \mathrm{Tl} \mathrm{ni} \mathrm{V}$ ), el tiempo de germinación tiene el orden siguiente: serrano $<$ jalapeño $<$ poblano, lo cual indica que las semillas de la variedad poblano tardaron mayor tiempo en germinar que las semillas de las otras dos variedades. Asimismo, los resultados muestran que la velocidad de germinación de las semillas de jalapeño no fue afectada por los tratamientos con $\mathrm{Cd}$ : el Tl la retrasó y el V la incrementó. La velocidad de germinación de las semillas de poblano no fue influenciada por la aplicación de $\mathrm{Cd}, \mathrm{Tl}$ o V. Por el contrario, la variedad serrano fue más sensible a los metales probados, al reducirse significativamente el CVG en presencia de éstos.

\section{Longitud de radícula}

Las plántulas de chile jalapeño y serrano tratadas con $\mathrm{Cd}$ no presentaron cambios significativos en la longitud de radícula en comparación con el testigo. Por el contrario, la longitud de la radícula de la variedad poblano fue severamente afectada por el tratamiento con $\mathrm{Cd}$, ya que los tratamientos con 25 y $50 \mu \mathrm{M} \mathrm{Cd}$ la redujeron en 46 y $38 \%$, respectivamente, en comparación con el testigo (Cuadro IV). En Albizia lebbeck, la aplicación de 10, 30, 50, 70 y $90 \mu \mathrm{mol} \mathrm{Cd} / \mathrm{L}$ provocaron reducción significativa del crecimiento de raíces, al compararlo con el grupo testigo. Esto puede deberse a la afectación del metabolismo celular de las raíces, lo que ocasiona una reducción en la formación de nuevas células y en la elongación de la región de extensión de la raíz (Farooqi et al. 2009).

Los tratamientos con $\mathrm{Tl}$ no afectaron de manera significativa la longitud de radícula en plántulas de la variedad poblano. Por otra parte, la radícula de plántulas de chile jalapeño mostró inhibición significativa de su crecimiento sólo con la concentración alta de $\mathrm{Tl}(50 \mu \mathrm{M})$, con decrementos del $66.2 \%$ respecto al testigo. La variedad serrano fue la más afectada por la aplicación de Tl, dado que se redujo significativamente el crecimiento de la radícula, lo cual fue dependiente de las dosis de $\mathrm{Tl}$ probadas, siendo éstas del orden de 56.6 y $67.7 \%$ con 25 y $50 \mu \mathrm{M} \mathrm{Tl}$, respectivamente, en comparación con el testigo (Cuadro IV). Este efecto inhibitorio se ha reportado en col, lechuga, mijo, rábano (Raphanus sativus), nabo y trigo tratados con soluciones de Tly $\mathrm{V}$ a concentraciones de 0 a $40 \mathrm{mg} / \mathrm{L}$, encontrándose que ambos elementos reducen la elongación de la raíz (Carlson et al. 1991). Asimismo se ha reportado que el $\mathrm{Tl}$ a concentración de $510 \mathrm{mg} / \mathrm{L}$ disminuye significativamente la longitud de la radícula y del eje embrionario de semillas de arroz (Yao et al. 2008).

Los tratamientos con V incrementaron la longitud de radícula en plántulas de las variedades jalapeño y serrano, aunque estos incrementos no fueron estadísticamente significativos. En el caso de la variedad 
poblano, se observaron efectos negativos del tratamiento con $\mathrm{V}$, al reducirse la longitud de la radícula en 33.3 y $52.3 \%$ cuando recibieron las dosis de 25 y $50 \mu \mathrm{M}$, respectivamente, en comparación con el testigo (Cuadro IV).

CUADRO IV. LONGITUD DE RADÍCULA (EN CENTÍMETROS) DE PLÁNTULAS PROVENIENTES DE SEMILLAS DE TRES VARIEDADES DE CHILE TRATADAS CON Cd, TI Y V

\begin{tabular}{|c|c|c|c|}
\hline \multirow{2}{*}{ Variedad } & \multicolumn{3}{|c|}{$\mathrm{Cd}(\mu \mathrm{M})$} \\
\hline & 0 & 25 & 50 \\
\hline Jalapeño & $0.692 \pm 0.021 \mathrm{a}$ & $0.633 \pm 0.014 \mathrm{a}$ & $0.733 \pm 0.068 \mathrm{a}$ \\
\hline Poblano & $0.525 \pm 0.008 \mathrm{a}$ & $0.283 \pm 0.032 \mathrm{~b}$ & $0.325 \pm 0.028 b$ \\
\hline Serrano & $0.825 \pm 0.075 \mathrm{a}$ & $0.767 \pm 0.076 \mathrm{a}$ & $0.767 \pm 0.076 \mathrm{a}$ \\
\hline \multirow{2}{*}{ Variedad } & \multicolumn{3}{|c|}{$\mathrm{Tl}(\mu \mathrm{M})$} \\
\hline & 0 & 25 & 50 \\
\hline Jalapeño & $0.692 \pm 0.021 \mathrm{a}$ & $0.747 \pm 0.019 \mathrm{a}$ & $0.233 \pm 0.014 \mathrm{~b}$ \\
\hline Poblano & $0.524 \pm 0.008 \mathrm{a}$ & $0.531 \pm 0.066 \mathrm{a}$ & $0.375 \pm 0.066 \mathrm{a}$ \\
\hline Serrano & $0.825 \pm 0.075 \mathrm{a}$ & $0.358 \pm 0.048 \mathrm{~b}$ & $0.267 \pm 0.024 b$ \\
\hline \multirow{2}{*}{ Variedad } & \multicolumn{3}{|c|}{$\mathrm{V}(\mu \mathrm{M})$} \\
\hline & 0 & 25 & 50 \\
\hline Jalapeño & $0.692 \pm 0.021 \mathrm{a}$ & $0.692 \pm 0.050 \mathrm{a}$ & $0.850 \pm 0.109 \mathrm{a}$ \\
\hline Poblano & $0.525 \pm 0.008 \mathrm{a}$ & $0.350 \pm 0.017 b$ & $0.250 \pm 0.040 \mathrm{c}$ \\
\hline Serrano & $0.825 \pm 0.075 \mathrm{a}$ & $0.842 \pm 0.102 \mathrm{a}$ & $0.958 \pm 0.109 \mathrm{a}$ \\
\hline
\end{tabular}

Medias \pm desviación estándar con letras distintas en cada hilera, indican que existen diferencias estadísticas significativas (diferencia mínima significativa, $\mathrm{p} \leq 0.05$ )

\section{Longitud de vástago}

El Cd no tuvo efecto en la longitud de vástago de las variedades jalapeño y serrano. De manera interesante, el crecimiento del vástago en la variedad poblano fue estimulado por la adición de $\mathrm{Cd}$ a dosis de 25 y $50 \mu \mathrm{M}$, en 67.7 y $96.8 \%$, respectivamente, en comparación con el testigo (Cuadro V).

El Tl afectó de manera diferencial la longitud de vástago en las tres variedades de chile probadas. En las variedades jalapeño y poblano, el tratamiento con $25 \mu \mathrm{M}$ Cd estimuló el crecimiento del vástago en 46.2 y $31.2 \%$, respectivamente, en comparación con sus correspondientes testigos. En contraste con lo anterior, se reporta que tratamientos de $10 \mathrm{a}$ $90 \mu \mathrm{mol} \mathrm{Cd} / \mathrm{L}$ afectan el crecimiento de las plántulas de Albizia lebbeck, en comparación con su grupo testigo (Farooqi et al. 2009). Por otra parte, tratamientos de $0.25 \mathrm{mM} \mathrm{Cd}$ inhiben significativamente el crecimiento de la plúmula de semillas de chícharo (Pisum sativum) (Chugh y Sawhney 1996).

En las dos concentraciones suministradas, el Tl redujo de manera significativa el crecimiento de la parte aérea de las plántulas de chile serrano; estas reducciones fueron de $61.2 \%$ con $25 \mu \mathrm{M}$ Tl y de $65.5 \%$ con $50 \mu \mathrm{M} \mathrm{Tl}$, en ambos casos respecto al testigo (Cuadro V). En pepino (Cucumis sativus), plántulas con deficiencia de potasio resultan más sensibles al tratamiento con $\mathrm{Tl}$, en tanto que la aplicación de $10 \mu \mathrm{M}$ Tl afectó la multiplicación celular de manera más evidente que la elongación y la diferenciación celular (Siegel y Siegel 1976).

CUADRO V. LONGITUD DE VÁSTAGO (EN CENTÍMETROS) DE PLÁNTULAS PROVENIENTES DE SEMILLAS DE TRES VARIEDADES DE CHILE TRATADAS CON Cd, Tl Y V

\begin{tabular}{lccc}
\hline \multirow{4}{*}{ Variedad } & \multicolumn{4}{c}{$\mathrm{Cd}(\mu \mathrm{M})$} \\
\cline { 2 - 4 } & 0 & 25 & 50 \\
\hline Jalapeño & $2.667 \pm 0.194 \mathrm{a}$ & $2.658 \pm 0.167 \mathrm{a}$ & $2.667 \pm 0.209 \mathrm{a}$ \\
Poblano & $1.033 \pm 0.163 \mathrm{~b}$ & $1.733 \pm 0.177 \mathrm{a}$ & $2.033 \pm 0.180 \mathrm{a}$ \\
Serrano & $5.092 \pm 0.119 \mathrm{a}$ & $4.667 \pm 0.221 \mathrm{a}$ & $4.667 \pm 0.221 \mathrm{a}$ \\
\hline \multirow{4}{*}{$\mathrm{Tl}(\mu \mathrm{M})$} \\
Variedad & 0 & 25 & 50
\end{tabular}

$\begin{array}{llll}\text { Jalapeño } & 2.667 \pm 0.194 \mathrm{~b} & 3.899 \pm 0.279 \mathrm{a} & 1.617 \pm 0.177 \mathrm{c}\end{array}$ Poblano $\quad 1.034 \pm 0.163 \mathrm{~b} \quad 2.406 \pm 0.418$ a $1.834 \pm 0.176 \mathrm{ab}$ $\begin{array}{llll}\text { Serrano } & 5.092 \pm 0.119 a & 1.975 \pm 0.160 b & 1.758 \pm 0.064 b\end{array}$

\begin{tabular}{lccc}
\hline \multirow{4}{*}{ Variedad } & \multicolumn{4}{c}{$\mathrm{V}(\mu \mathrm{M})$} \\
\cline { 2 - 4 } & 0 & 25 & 50 \\
\hline Jalapeño & $2.667 \pm 0.194 \mathrm{a}$ & $3.058 \pm 0.317 \mathrm{a}$ & $2.992 \pm 0.121 \mathrm{a}$ \\
Poblano & $1.034 \pm 0.163 \mathrm{~b}$ & $2.367 \pm 0.261 \mathrm{a}$ & $1.284 \pm 0183 \mathrm{~b}$ \\
Serrano & $5.092 \pm 0.119 \mathrm{a}$ & $4.458 \pm 0.355 \mathrm{a}$ & $3.958 \pm 0.645 \mathrm{a}$
\end{tabular}

Medias \pm desviación estándar con letras distintas en cada hilera, indican que existen diferencias estadísticas significativas (diferencia mínima significativa, $\mathrm{p} \leq 0.05$ )

En las semillas tratadas con V, no se encontraron diferencias significativas en la longitud de vástago de las variedades jalapeño y serrano. Por el contrario, en chile poblano, a la dosis de $25 \mu \mathrm{M}$ la longitud de vástago fue mayor en $56.3 \%$ a la registrada en el testigo, y superior en $45.8 \%$ al tratamiento con $50 \mu \mathrm{M}$ V (Cuadro V).

\section{Crecimiento relativo de la radícula $(C R R)$}

El suministro de $\mathrm{Cd}$ a ambas dosis probadas redujo de manera significativa el crecimiento relativo de 
la radícula sólo en la variedad poblano. En promedio, el CRR con los tratamientos con 25 y $50 \mu \mathrm{M} \mathrm{Cd}$ fue de $57.9 \%$ respecto al testigo (Fig. 3a).

En la variedad jalapeño, el CRR fue menor al $40 \%$ con el tratamiento con $50 \mu \mathrm{M} \mathrm{Tl}$, mientras que en la variedad serrano, con la aplicación de 25 y $50 \mu \mathrm{M}$ $\mathrm{Tl}$ los valores de CRR fueron menores al 50 y $40 \%$, respectivamente. En la variedad poblano se observa que el $\mathrm{Tl}$ reduce ligeramente el CRR, pero dichas reducciones no son significativas (Fig. 3b).

En semillas tratadas con V, solamente se observaron diferencias estadísticas significativas en el valor del CRR con la variedad poblano, registrándose decrementos del 33.3 y $52.3 \%$ a las dosis de 25 y $50 \mu \mathrm{M}$, respectivamente; lo anterior indica que esta variedad es más susceptible a los efectos fitotóxicos del V(Fig. 3c).

\section{CONCLUSIONES}

Los elementos $\mathrm{Cd}$, $\mathrm{Tl}$ y $\mathrm{V}$ producen efectos diferenciales en la germinación de semillas y en el crecimiento inicial de las plántulas de chile. Tales diferencias son atribuibles a las variedades de chile evaluadas, las concentraciones de metales probadas y las variables medidas.

El PG y el PRG no fueron afectados por las concentraciones de $\mathrm{Cd}$, $\mathrm{Tl}$ y $\mathrm{V}$ suministradas. Por el contrario, los efectos de estos elementos fueron observados en indicadores relacionados con el crecimiento inicial de la plántula.

Al comparar las respuestas de las variedades respecto a sus testigos correspondientes, se pudo observar que el orden de tolerancia de las variedades de chile evaluadas es jalapeño $>$ poblano $>$ serrano. En este caso, la mayor tolerancia mostrada por el chile jalapeño corresponde a una menor variación de las variables evaluadas en comparación con su testigo, en respuesta a los metales probados.

En el chile serrano los elementos probados afectaron negativamente las variables evaluadas, en tanto que en el chile jalapeño (el más tolerante a los elementos probados) el $\mathrm{Tl}$ fue el elemento que afectó el mayor número de variables evaluadas de manera negativa.

Efectos positivos de las concentraciones de $\mathrm{Cd}$ estudiadas se presentaron en la longitud de vástago de chile poblano. Asimismo, el Tl en las dosis ensayadas incrementó la longitud de vástago en las variedades jalapeño y poblano. Por otra parte, efectos positivos del V se observaron en el coeficiente de velocidad de germinación del chile jalapeño, así como en la longitud de vástago del chile poblano.
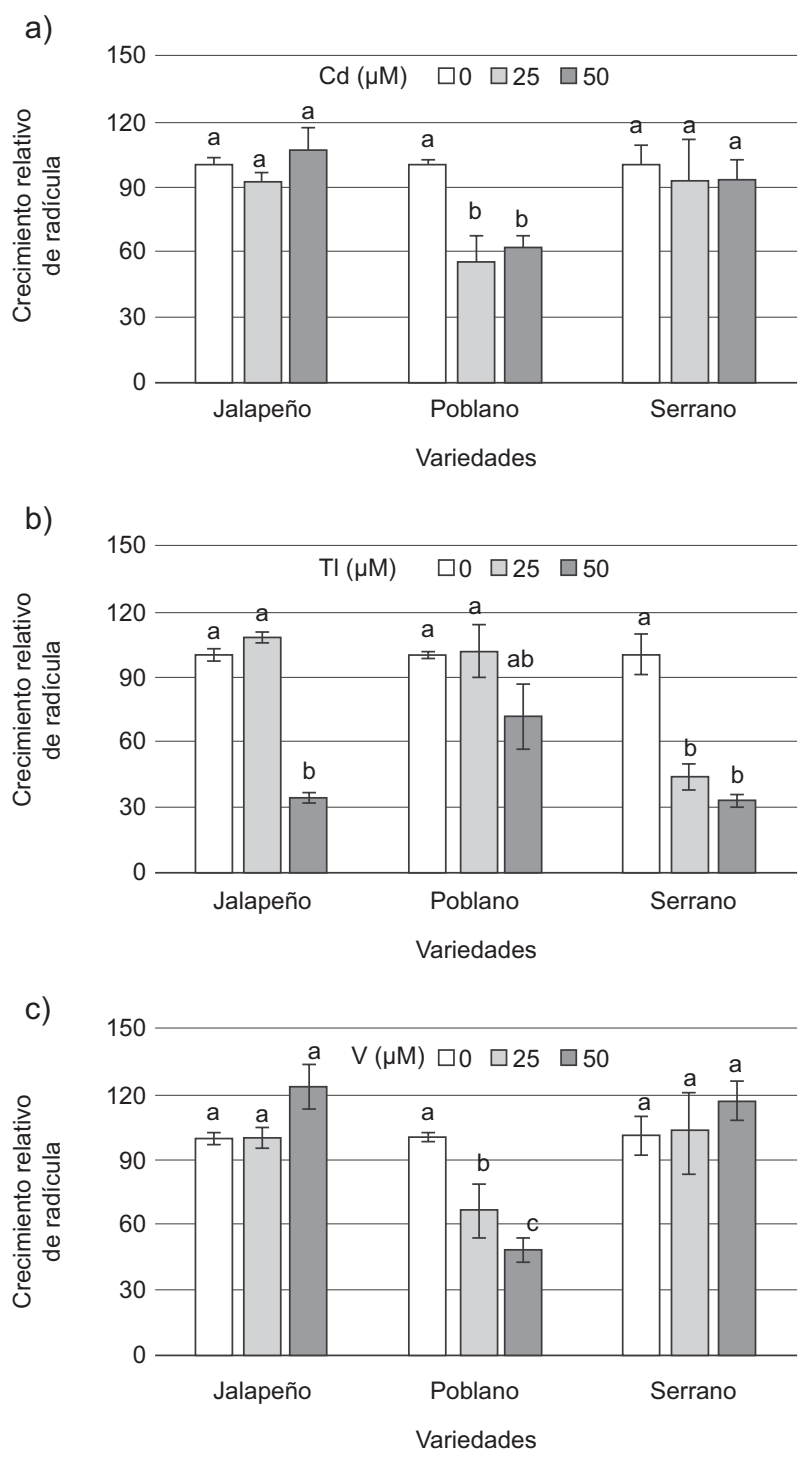

Fig. 3. Crecimiento relativo de radícula (CRR) de plántulas obtenidas de semillas de tres variedades de chile tratadas con (a) Cd, (b) Tl y (c) V. Medias \pm desviación estándar con letras distintas en cada variedad por subfigura indican que existen diferencias estadísticas significativas (Diferencia mínima significativa, $\mathrm{p} \leq 0.05$ )

\section{REFERENCIAS}

Ahmad I., Akhtar M.J., Zahir Z.A. y Jamil A. (2012). Effect of cadmium on seed germination and seedling growth of four wheat (Triticum aestivum L.) cultivars. Pak. J. Bot. 44 (5), 1569-1574.

Aina R., Labra M., Fumagalli P., Vannini C., Marsoni M., Cucchi U., Bracale M., Sgorbati S. y Citterio S. (2007). Thiol-peptide level and proteomic changes in response 
to cadmium toxicity in Oryza sativa L. roots. Environ. Exp. Bot. 59 (3), 381-392.

DOI: $10.1016 /$ j.envexpbot.2006.04.010

Alcalá J., Sosa M., Moreno M., Rodríguez J.C., Quintana C., Terrazas C. y Rivero O. (2009). Metales pesados en suelo urbano como un indicador de la calidad ambiental: ciudad de Chihuahua, México. Multequina 18 (2), 53-69.

Aloui H., Souguir M. y Hannachi C. (2014). Determination of an optimal priming duration and concentration protocol for pepper seeds (Capsicum annuиm L.). Acta Agric. Slov. 103 (2), 213-221.

DOI: 10.14720 /aas.2014.103.2.6

Aslam R., Ansari M.Y.K., Choudhary S., Bhat T.M. y Jahan N. (2014). Genotoxic effects of heavy metal cadmium on growth, biochemical, cyto-physiological parameters and detection of DNA polymorphism by RAPD in Capsicum annuum L. - An important spice crop of India. Saudi J. Biol. Sci. 21 (5), 465-472.

DOI: 10.1016/j.sjbs.2014.07.005

Ayala-Villegas M.J., Ayala-Garay O.J., Aguilar-Rincón V.H. y Corona-Torres T. (2014). Evolución de la calidad de semilla de Capsicum annuиm L. durante su desarrollo en el fruto. Rev. Fitotec. Mex. 37 (1), 79-87.

Babić M., Radić S., Cvjetko P., Roje V., Pevalek-Kozlina B. y Pavlica M. (2009). Antioxidative response of Lemna minor plants exposed to thallium (I)-acetate. Aquat. Bot. 91 (3), 166-172.

DOI: 10.1016/j.aquabot.2009.05.005

Bautista V.O., Fischer G. y Cárdenas J.F. (2013). Cadmium and chromium effects on seed germination and root elongation in lettuce, spinach and Swiss chard. Agron. Colomb. 31 (1), 48-57.

Carlson C.L., Adriano D.C., Sajwan K.S., Abels S.L., Thoma D.P. y Driver J.T. (1991). Effects of selected trace metals on germinating seeds of six plant species. Water Air Soil Poll. 59 (3-4), 231-240.

DOI: $10.1007 / \mathrm{BF} 00211832$

Chugh L.K. y Sawhney S.K. (1996). Effect of cadmium on germination, amylases and rate of respiration of germinating pea seeds. Environ. Pollut. 92 (1), 1-5. DOI: 10.1016/0269-7491(95)00093-3

Demir I., Ermis S., Mavi K. y Matthews S. (2008). Mean germination time of pepper seed lots (Capsicum annuит L.) predicts size and uniformity of seedlings in germination tests and transplant modules. Seed Sci. Technol. 36 (1), 21-30.

DOI: $10.15258 /$ sst.2008.36.1.02

Di Candito M., Ranalli P. y Dal Re L. (2004). Heavy metal tolerance and uptake of $\mathrm{Cd}, \mathrm{Pb}$ and $\mathrm{Tl}$ by hemp. Adv. Hortic. Sci. 18 (3), 138-144.

Emino E.R. y Warman P.R. (2004). Biological assay for compost quality. Compost Sci. Util. 12 (4), 342-348. DOI: 10.1080/1065657X.2004.10702203
Farooqi Z.R., Iqbal M.Z., Kabir M. y Shafiq M. (2009). Toxic effects of lead and cadmium on germination and seedling growth of Albizia lebbeck (L.) Benth. Pak. J. Bot. 41 (1), 27-33.

Frías-Espericueta M.G., Osuna-López I., Bañuelos-Vargas I., López-López G., Muy-Rangel M.D., IzaguirreFierro G., Rubio-Carrasco W., Meza-Guerrero P.C. y Voltolina D. (2009). Cadmium, copper, lead and zinc contents of the mangrove oyster, Crassostrea corteziensis, of seven coastal lagoons of NW Mexico. Bull. Environ. Contam. Toxicol. 83 (4): 595-599. DOI: $10.1007 / \mathrm{s} 00128-009-9828-\mathrm{z}$

Garay-Hernández M.D.C., Paredes-Lara M.R. y Hernández-Martínez J. (2012). Acumulación de As-Cd-Pb en suelos y cultivos afectados por jales mineros. Memorias. V Congreso de la Asociación Mesoamericana de Ecotoxicología y Química Ambiental. Aguascalientes, Ags., 25 al 28 de julio, pp. 1-10.

García-Gaytán V., Gómez-Merino F.C., Trejo-Téllez L.I., Baca-Castillo G.A. y García-Morales S. (2017). The chilhuacle chili (Capsicum annuum L.) in Mexico: Description of the variety, its cultivation, and uses. Int. J. Agron. 2017, article ID 5641680.

DOI: $10.1155 / 2017 / 5641680$

González-Dávila O., Gómez-Bernal J.M. y Ruiz-Huerta E.A. (2012). Plants and soil contamination with heavy metals in agricultural areas of Guadalupe, Zacatecas, Mexico. En: Environmental contamination (Srivastava J.K., Ed.). InTech, Rijeka, Croacia, pp. 37-50. DOI: $10.5772 / 31062$

He J., Ren Y., Chen X. y Chen H. (2014). Protective roles of nitric oxide on seed germination and seedling growth of rice (Oryza sativa L.) under cadmium stress. Ecotoxicol. Environ. Saf. 108, 114-119.

DOI: 10.1016/j.ecoenv.2014.05.021

Herrera J., Alizaga R., Guevara E., Jiménez V., Mora F.N., Badilla J.R. y Salazar W. (2006). Germinación y crecimiento de la planta. En: Fisiología de la producción de los cultivos tropicales. (Villalobos-Rodríguez E., Ed.). Universidad de Costa Rica, San José, Costa Rica, vol. 4, pp. 1-45.

Iannacone O.J. y Alvariño F.L. (2005). Efecto ecotoxicológico de tres metales pesados sobre el crecimiento radicular de cuatro plantas vasculares. Agric. Téc. 65 (2), 198-203. DOI: $10.4067 / \mathrm{S} 0365-28072005000200009$

Idrees S., Shabir S., Ilyas N., Batool N. y Kanwal S. (2015). Assessement of cadmium on wheat (Triticum aestivum L.) in hydroponics medium. Agrociencia 49 (8), 917-929.

ISTA (2009). International rules for seed testing. Seed Sciences and Techonology, International Seed Testing Association, Bassersdorf, Suiza, 27 pp. 
Kader M.A. (2005). A comparison of seed germination calculation formulae and the associated interpretation of resulting data. J. Proc. Royal Soc. New South Wales 138, 65-75.

Kasai M., Yamazaki J., Kikuchi M., Iwaya M. y Sawada S. (1999). Concentration of vanadium in soil water and its effect on growth and metabolism of rye and wheat plants. Commun. Soil Sci. Plant Anal. 30 (7-8), 971982. DOI: $10.1080 / 00103629909370261$

Khan S.U. y Moheman A. (2006). Effect of heavy metals (cadmium and nickel) on the seed germination, growth and metals uptake by chilli (Capsicum frutescens) and sunflower plants (Helianthus annuus). Pollut. Res. 25 (1), 99-104.

Kranner I. y Colville L. (2011). Metals and seeds: Biochemical and molecular implications and their significance for seed germination. Environ. Exp. Bot. 72 (1), 93-105. DOI: 10.1016/j.envexpbot.2010.05.005

Kumar S. (2017). Sphagnum moss as a growing media constituent: some effects of harvesting, processing and storage. Mires and Peat. 20 (2017/18), article 07, 1-11. DOI: 10.19189/MaP.2016.OMB.232

Lee S.J., Park J.H., Ahn Y.T. y Chung J.W. (2015). Comparison of heavy metal adsorption by peat moss and peat moss-derived biochar produced under different carbonization conditions. Water Air Soil Pollut. 226, 9. DOI: $10.1007 / \mathrm{s} 11270-014-2275-4$

Liu S., Yang C., Xie W., Xia Ch. y Fan P. (2012). The effects of cadmium on germination and seedling growth of Suaeda salsa. Procedia Environ. Sci. 16 (2012), 293-298. DOI: 10.1016/j.proenv.2012.10.041

Maldonado V.M., Rubio Arias H.O., Quintana R., Saucedo R.A., Gutiérrez M., Ortega J.A. y Nevarez G.V. (2008). Heavy metal content in soils under different wastewater irrigation patterns in Chihuahua, Mexico. Int. J. Environ. Res. Public Health 5 (5), 441-449.

DOI: $10.3390 /$ ijerph5050441

Meyer C., Díaz-de-Quijano M., Monna F., Franchi M., Toussaint M.L., Gilbert D. y Bernard N. (2015). Characterisation and distribution of deposited trace elements transported over long and intermediate distances in north-eastern France using Sphagnum peatlands as a sentinel ecosystem. Atmos. Environ. 101, 286-293. DOI: 10.1016/j.atmosenv.2014.11.041

Navarro A.J.P., Alonso I.A. y López M.J.R. (2007). Aspectos bioquímicos y genéticos de la tolerancia $\mathrm{y}$ acumulación de metales pesados en plantas. Rev. Ecosist. 16 (2), 10-25.

Queirolo F., Stegen S., Contreras-Ortega C., Ostapczuk P., Queirolo A. y Paredes B. (2009). Thallium levels and bioaccumulation in environmental samples of northern Chile: Human health risks. J. Chil. Chem. Soc. 54 (4), 464-469. DOI: 10.4067/S0717-97072009000400031
Ranal M.A. y García D.S.D. (2006). How and why to measure the germination process? Braz. J. Bot. 29 (1), 1-11. DOI: 10.1590/S0100-84042006000100002

SAGARPA (2015). Producción de chile mexicano. Secretaría de Agricultura, Ganadería, Desarrollo Rural, Pesca y Alimentación, México [en línea]. http:// www.gob.mx/sagarpa/articulos/produccion-del-chilemexicano 28/02/2017

Sánchez J.A., Montejo L., Gamboa A., Albert P.D. y Hernández F. (2015). Germinación y dormancia de arbustos y trepadoras del bosque siempreverde de la Sierra del Rosario, Cuba. Pastos y Forrajes 38 (1), 11-28.

SAS (2011). Base SAS $^{\circledR} 9.3$ procedures guide: Statistical procedures. SAS Institute, Cary, N.C., EUA, 536 pp.

SEMARNAT (2007). Norma Oficial Mexicana NOM147-SEMARNAT/SSA1-2004. Que establece criterios para determinar las concentraciones de remediación de suelos contaminados por arsénico, bario, berilio, cadmio, cromo hexavalente, mercurio, níquel, plata, plomo, selenio, talio y/o vanadio. Secretaría de Medio Ambiente y Recursos Naturales. Diario Oficial de la Federación, 2 de marzo.

Siegel B.Z. y Siegel S.M. (1976). Effect of potassium on thallium toxicity in cucumber seedlings: Further evidence for potassium-thallium ion antagonism. Bioinorg. Chem. 6 (4), 341-345.

DOI: $10.1016 / \mathrm{S} 0006-3061(00) 80019-8$

Sobrero M.C. y Ronco A. (2004). Ensayo de toxicidad aguda con semillas de lechuga (Lactuca sativa L.). En: Ensayos toxicológicos y métodos de evaluación de calidad de aguas. Estandarización, intercalibración, resultados y aplicaciones (Castillo G., Ed.). Centro Internacional de Investigaciones para el Desarrollo, Instituto Mexicano de Tecnología del Agua, Ottawa, Canadá, pp. 71-79.

Sobrevilla-Solís J.A., López-Herrera M., López-Escamilla A.L. y Romero-Bautista L. (2013). Evaluación de diferentes tratamientos pregerminativo y osmóticos en la germinación de semillas Prosopis laevigata (Humb. y Bonpl. Ex Willd) M.C. Johnston. En: Estudios científicos en el estado de Hidalgo y zonas aledañas. (PulidoFlores G. y Monks S., Eds.). Zea Books, Lincoln, NE, EUA, vol. II, pp. 83-95.

Tangahu B.V., Sheikh A.S.R., Basri H., Idris M., Anuar N. y Mukhlisin M. (2011). A review on heavy metals (As, $\mathrm{Pb}$, and $\mathrm{Hg}$ ) uptake by plants through phytoremediation. Int. J. Chem. Eng. 2011, article ID 939161. DOI: $10.1155 / 2011 / 939161$

Tchounwou P.B., Yedjou C.G., Patlolla A.K. y Sutton D.J. (2012). Heavy metals toxicity and the environment. Mol. Clin. Environ. Toxicol. EXS 101, 133-164. DOI: 10.1007/978-3-7643-8340-4_6 
Tham L.X., Nagasawa N., Matsuhashi S., Ishioka N.S., Ito T. y Kume T. (2001). Effect of radiation-degraded chitosan on plants stressed with vanadium. Radiat. Phys. Chem. 61 (2), 171-175. DOI: $10.1016 / \mathrm{S} 0969-806 \mathrm{X}(00) 00388-1$

Tiquia S.M. (2000). Evaluating phytotoxicity of pig manure from the pig-on-litter system. Proceedings. International Composting Symposium (ICS'99) vols. 1 y 2. Halifax/Dartmouth, Nueva Escocia, Canadá, septiembre 19-23, pp. 625-647.

Walter I., Martínez F. y Cala V. (2006). Heavy metal speciation and phytotoxic effects of three representative sewage sludges for agricultural uses. Environ. Pollut. 139 (3), 507-514.

DOI: 10.1016/j.envpol.2005.05.020
Wang X.F. y Zhou Q.X. (2005). Ecotoxicological effects of cadmium on three ornamentals plants. Chemosphere 60 (1), 16-21. DOI: 10.1016/j.chemosphere.2004.12.031

Yao Y., Zhang P., Chen X.Y., Chen J., Wu T. y Chen Y.H. (2008). Effects of thallium on seed germination and plant growth of Oryza sativa L. Acta Agr. Bor.-Sin. 23 (1), 138-140. DOI: 10.7668/hbnxb.2008.01.030

Zhang L., Mao X. y Xia Z. (2012). Effects of sodium metavanadate and germination on the sprouting of chickpeas and its content of vanadium, formononetin and biochanin a in the sprouts. J. Diet. Suppl. 9 (1), 34-44. DOI: 10.3109/19390211.2011.639858

Zhao T., Tie B.-Q. y Yang S.-W. (2007). Effect of vanadium on seed germination and seedling growth. Environ. Sci. Technol. 30 (5), 3-5. 